\section{Old Botanical Techniques for New Microscopes}

\author{
Jim Haseloff \\ University of Cambridge, Cambridge, UK \\ BioTechniques 34:1174-1182 (June 2003)
}

\section{CLASSICAL BOTANICAL MICROSCOPY}

Plant specimens have been used for microscopy since the first construction of suitable optical instruments because they provided a convenient source of material that was easy to section and prepare for microscopy. The walls of plant cells are prominent and, consequently, observations of plants have played a crucial role in the development of key cell biology concepts. The English scientist, Robert Hooke (1635-1702), built one of the earliest compound microscopes and published the first monograph devoted to microscopy. In this 1667 work, Micrographica (1), he described the microscopic compartments formed from the thickened walls that made up the structure of a slice of cork. He called these compartments "cells" after the Latin words "cella," meaning small room or cubicle, and its derivative "cellulae," which was applied to the hexagonal cells of a honeycomb. Although Hooke's view of cells was different from our modern understanding (he believed them to be passages for the flow of sap through the plant), his work provided a basis for the identification of different cell types and tissue organization in plants by other scientists.

Nehemiah Grew (1641-1712), in England, and his contemporary, Marcello Malphigi (1628-1694), in Italy, were the co-founders of plant anatomy. Both had medical training and used microscopy to investigate plant structure. They invented comparative anatomy and were the first to describe vegetable structures as being built from tissues that contained particular cells. In a series of publications from 1670 through 1682, Grew established that plant organs were formed from two distinct tissues, composed of either fibers or pithy parts composed of parenchyma (a term invented by Grew). Grew also invented the term "tissue," based on a misinterpretation of cell wall structure. He believed that the scaffolding of cell walls consisted of fine threads and that plant organization resembled a tissue of lace-like layers. Despite this misapprehension, the term caught on and was even adopted by animal histologists. Grew described the structure plant organs involved in reproduction and germination. Malphigi published Anatomia plantarum in 1679, in which he described the inner structure of roots, the sap containing fibers of wood, and stomata. However, there were few important advances on the ideas of Grew and Malphigi for the next century.

\section{CELL THEORY}

By the late 1820s, improved microscopes with higher magnification, clearer visual fields, and corrected chromatic aberration became available. New composite lenses were constructed from glass with different indices of refraction, which allowed one lens component to cancel the chromatic aberrations introduced by the other. These achromatic lenses eliminated the colored fringes that plagued earlier microscopes and produced much sharper images. The use of the new microscopes triggered a series of advances over the next two decades. Again, the study of plant material was at the forefront of this emerging science.

It was a botanist, Robert Brown (1773-1858), who named the nucleus and recognized it as an essential constituent of living cells. In 1838, the botanist Mattias Jakob Schleiden (1804-1881) postulated that plant structure is composed entirely of cells or their products. Within a few months, his friend and colleague, Theodor Schwann (1810-1882), had proposed a similar conclusion for animal cells. Schleiden and Schwann received credit for the formulation of cell theory, but their views of the origin of cells were incorrect. It was the work of Dumortier with algae and Remak with animal cells that demonstrated that cells are formed by the scission of existing cells $(2,3)$. Thus, it was believed that tissues were formed by the proliferation of existing cells, although the details of nuclear division were still unknown. Cell theory emphasized the unity of living systems and provided a simple underpinning for many biological problems (4).

\section{SYNTHETIC DYES}

With the acceptance of the cell theory, attention focused on the constituents of cells. Cells were known to be composed of a wall or membrane that enclosed a viscous protoplasm and nucleus. Histological technique was still at a primitive stage in these early microscopy studies. Each worker would generally construct their own microscope and other equipment. Stains were rarely used. Plant specimens were sectioned by hand or dissected with needles and mounted in watery, low refractive index media. By the mid-1800s, natural dyes such as carmine were commonly being used to stain biological samples. In 1856, an 18-year-old chemistry assistant, William Henry Perkin, undertook a project to synthesize the antimalarial drug, quinine, during his Easter vacation. He started his synthesis with aniline, a waste product from coal tar. Unfortunately, his reactions failed to produce quinine but, instead, produced a vivid purple pigment, which he showed could be used as a colorfast mauve dye for fabrics. This discovery made Perkin a rich man and marked the start of the synthetic dye industry. Many hundreds of dyes with a wide range of properties would become available over the next 50 years. Microscopists rapidly adopted these new dyes from the textile industry and used them as cytological stains to provide selective coloring and higher contrast to their samples (5).

Parallel developments in specimen preparation resulted in the adoption of new fixatives, such as mercuric chloride (1856), osmium tetroxide (1864), and formaldehyde (1892). Concerted attempts were made to improve the sectioning of biological material. The first practical microtomes for cutting histological sections became available after the work of Wilhelm His (1870), and paraffin embedding was introduced by 
Frédericq (1879) and Otto Buschli (1881). Meanwhile, Carl Zeiss had quit his botanical studies with Schleiden and, with Ernst Abbe, designed new microscopes (1872) with a series of lenses that were corrected for chromatic aberration at three wavelengths across the visible spectrum and were also corrected for spherical aberration. Their high-power apochromatic objectives were designed for use with immersion oil, and their resolution approached the theoretical limit for light microscopy. These improved microscopes further spurred the development of improved histological technique.

By the end of the 19th century, techniques for the staining, thin sectioning, and magnification of biological tissues had reached a high art, and a succession of discoveries had resulted in the description of the main subcellular constituent of cells, including chromosomes, Golgi bodies, endoplasmic reticulum and mitochondria, observation of the dynamics of cell division, and the description of numerous, specialized cell types and tissues in animal and plant systems. However, optical microscopes provided few tools to probe these new systems. Instead, biochemistry, structural biology, and, lately, molecular genetics provided the primary tools of choice for biologists through most of the 20th century. Optical microscopy techniques remained important but were no longer at center stage. However, a major resurgence of interest in optical microscopy is underway.

\section{CONFOCAL LASER-SCANNING MICROSCOPY}

The development of the first practical confocal laser-scan-

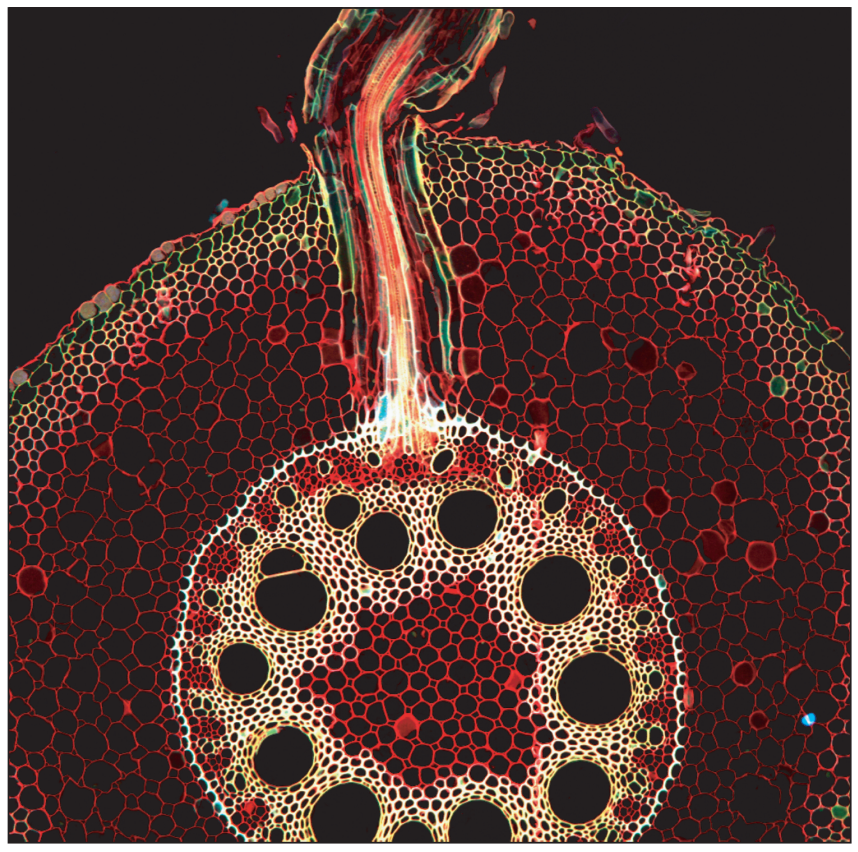

Figure 1. Confocal microscopy of a transverse section of a maize root. A Zea mays root was fixed, cleared in xylene, embedded in paraffin, sectioned using a microtome, and stained with Safranin O and Fast Green FCF. A digital image was collected using a Leica SP confocal microscope (Leica Microsystems Semiconductor GmbH, Wetzlar, Germany) with laser excitation at 488,568 , and $633 \mathrm{~nm}$. 


\section{Biolmaging}

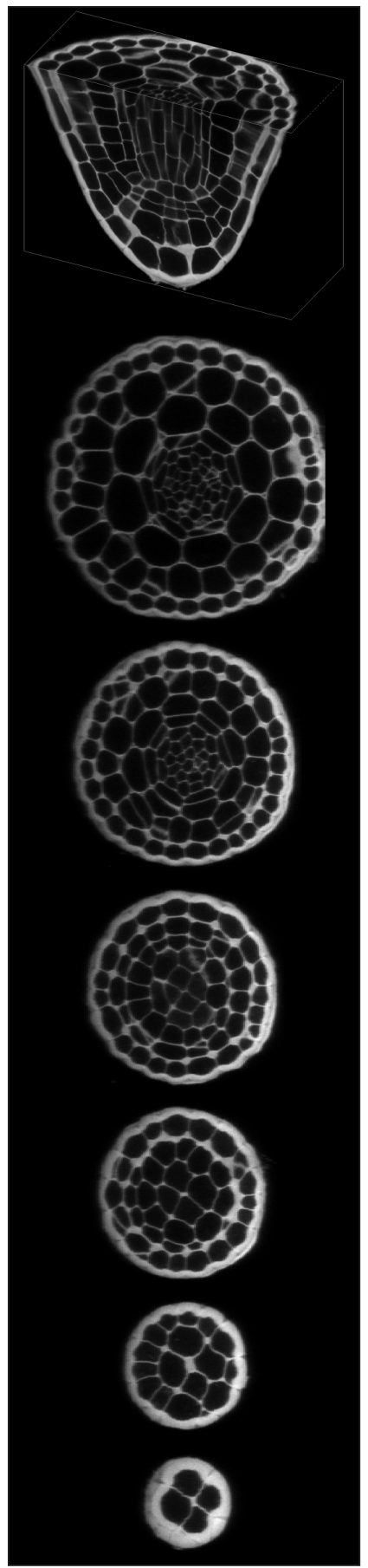

Figure 2. Chemical staining and deep optical sectioning of cleared Arabidopsis root tissues. Mature Arabidopsis embryos were stained using a pseudo-Schiff reaction to specifically label carbohydrate groups in cell walls. The tissues were mounted in a chloral hydrate-based clearing agent for microscopy. The combination of clearing and intense staining allows for deep optical sectioning of an entire embryo. Optical sections from the root meristem are shown. ning microscope (6) was a major advance in optical microscopy. These microscopes rely on a laser light source, which is scanned as a focused spot across the sample. Any fluorescence emission or reflected light that originates from the illuminated point of focus is directed by the optics through a confocal aperture to a detector. Unwanted, out-of-focus, and scattered light is largely excluded by the aperture. These microscopes allow researchers to obtain optical sections from intact specimens up to a depth of several hundred microns. A series of optical sections can be collected and used to build a computer-reconstructed 3-D view of the sample. The microscopes are now widely available and used in biology for the imaging of immunofluorescent labels, fluorochromes, and autofluorescent proteins.

\section{STAINS FOR PLANT HISTOLOGY AND CYTOLOGY}

A wide variety of staining techniques has been adopted for plant specimens over the past 150 years. Perhaps the most widely used general tissue stains are Safranin $\mathrm{O}$ and haematoxylin. These are often accompanied by the use of counterstains such as Fast Green, Orange G, or Alcian Blue. In addition, there is a large variety of more specific staining techniques that have been developed for particular plant materials and organelles. For example, Feulgen staining has been used for the specific labeling of DNA, the periodic acidSchiff reaction can be used to label carbohydrates, Aniline Blue for callose, Nile
Red for oil bodies, and Phloroglucinol for lignin. A multitude of published protocols are available. An excellent, recently published source of procedures can be found in Plant Microtechnique and Microscopy by Steven Ruzin (7).

Interestingly, many of the synthetic dyes used for plant microtechniques are highly fluorescent. This is particularly true for red, orange, and yellow dyes in the azine (e.g., Safranin O), acridine (e.g., Acridine Orange), and xanthene (e.g., Rhodamine) families. Thus, many classical histological techniques unintentionally produce specimens that are intensely fluorescent. In addition, aldehyde fixation, certain mountants, and long-term storage of stained preparations can result in tissue fluorescence, and the high concentration of stains deposited in the sections can lead to metachromasia (8). In our hands, it is rare to find stained and sectioned botanical material that is not highly fluorescent. The digital controls of a confocal microscope allow for the clean separation of different fluorescent emission signals and the balancing of signal levels in different channels. Thus, fluorescent images of exceptional clarity and vivid color can be easily obtained (Figure 1). In addition, the optical sectioning properties of the confocal microscope can be used to collect clear images from within thick sections and wholemounts.

\section{OPTICAL SECTIONING OF CLEARED WHOLE- MOUNTS}

It is difficult to observe cellular details that are deep in live plant tissue. Any light that penetrates the tissue must pass through many layers of cytoplasm, watery vacuole, and highly refractile cell walls. The different refractive indices of the material contribute to spherical aberration, and particulate subcellular matter also causes light scattering. Nomarski optics do not provide sufficient contrast and resolution to allow for the precise 3-D reconstruction of cell arrangements. To date, it has been difficult to obtain deep optical sections $(>50-100 \mu \mathrm{m})$ from intact tissue using confocal laser-scanning microscopy techniques. Rather, the 3-D anatomical arrangements of plant cells have conventionally been observed using microtomy techniques. However, the laborious nature of thin sectioning, the problem of obtaining the desired plane of the section, and difficulty of obtaining a complete series of sections have somewhat limited the use of microtomy techniques to the skilled and patient. Optical sectioning has many advantages from the point of view of speed and simplicity, and it can allow for the direct viewing of living wholemounts. Transverse sections would need to be reconstructed from a series of z-axis images.

The adoption of improved oil immersion objectives in the late 1800 s led to the development of clearing techniques. The aim was to infiltrate specimens before observation with a medium, which possesses a high index of refraction. This would produce a sample with glass-like optical properties and maximize the resolution available with the newly improved microscope optics. Clearing agents such as xylene, clove oil, cedar oil, and chloral hydrate were adopted and combined with compatible mountants such as Canada balsam and Hoyer's solution. All of these reagents have high refractive index similar 


\section{Biolmaging}

to that of glass (approximately 1.55). As a result of these clearing treatments, tissue sections generally become transparent, which greatly reduces problems with light scattering and spherical aberration. This allows for high-resolution imaging of thin sections (Figure 1). When the same techniques are applied to thick sections or wholemounts, the results of clearing are even more startling. However, the stain or fluorochrome is generally distributed throughout the cleared tissue, and details deep in the sample are still obscured by overlying signal. Here the confocal laser-scanning microscope proves its worth. High-resolution optical sections can be collected deep into such cleared samples to distances of greater than $200 \mu \mathrm{m}$. The depth of image collection is limited mainly by the working distance of available high numerical aperture objectives. The application of classical highly fluorescent stains and clearing techniques creates a new field of opportunities for modern confocal microscopy and computing methods.

We are interested in visualizing 3-D cell arrangements within the model plant, Arabidopsis thaliana. We have delved into classical plant microtechnique and devised several new techniques for intense, specific staining and clearing of plant architecture for 3-D microscopy (9). For example, the periodic acid treatment of carbohydrates produces aldehyde groups that can be reacted with various fluorescent pseudo-Schiff reagents. If fixed plant tissue is treated in this way, then cell walls (and starch-containing plastids, if present) become intensely and covalently labeled with the fluor. The tissue can then be directly cleared in a high refractive index agent containing chloral hydrate and mounted in Hoyer's solution for microscopy (Haseloff, Marrison, Wenzel, and Bougourd, unpublished results). The combination of high levels of fluorescence and high refractive index mountant allows for the collection of extended z-series images at very fine resolution (0.1-0.5 $\mu \mathrm{M}$ steps), using minimum confocal aperture and without fear of photobleaching or signal and resolution loss due to spherical aberration. Imaging to a depth of around 200 $\mu \mathrm{M}$ allows simple optical sectioning throughout an entire Arabidopsis root at high resolution. In fact, every cell within a mature Arabidopsis embryo can be clearly visualized (Figures 2 and $3 \mathrm{~A})$.
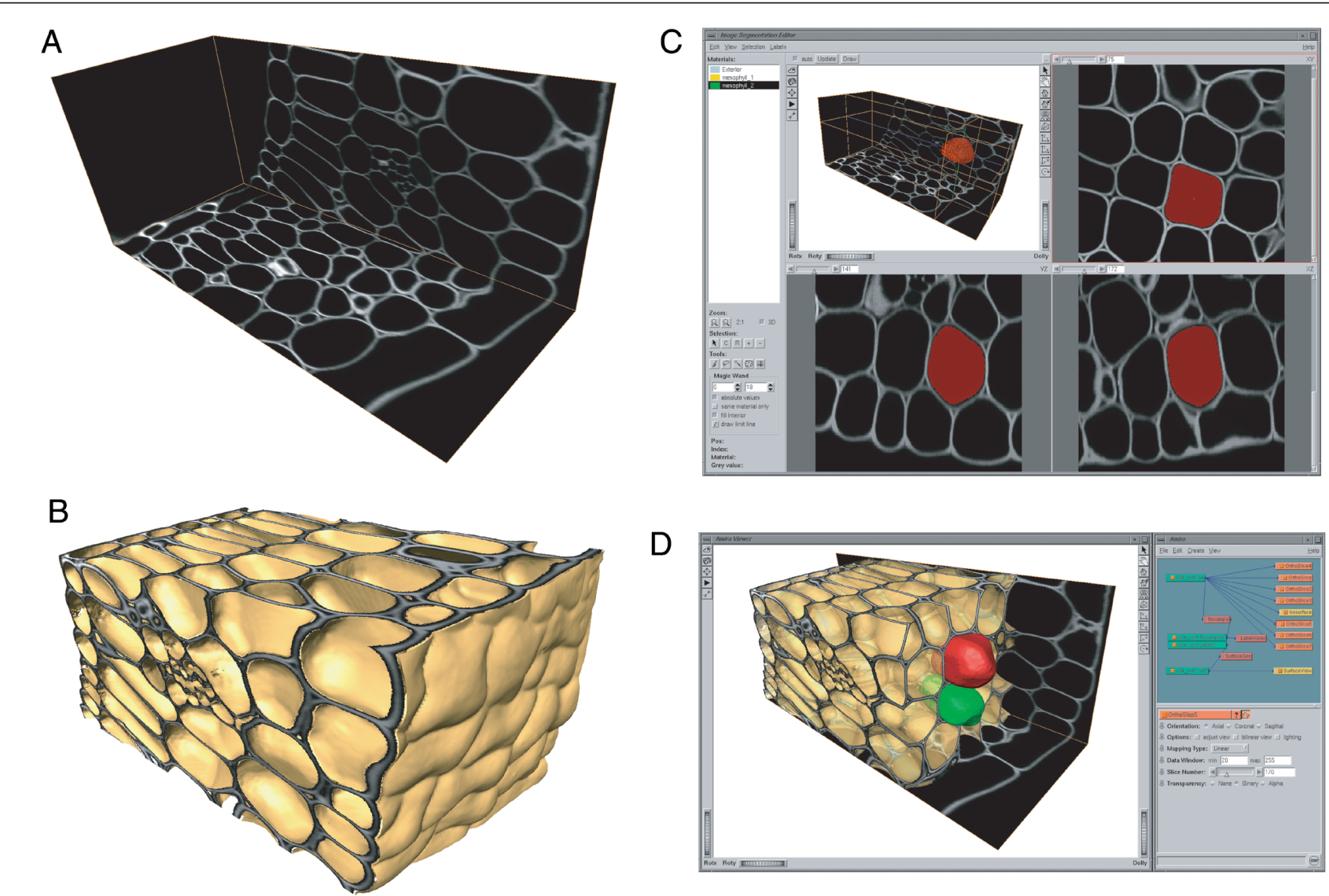

Figure 3. The 3-D segmentation of plant cells. (A) In the first step, a series of confocal optical sections, corresponding to a segment of cotyledon from an Arabidopsis embryo, is loaded into the Amira software. The software package provides modules for data visualization, including orthogonal slicing routines. (B) Modules are also available for the surface mapping, segmentation of volumes, and rendering of surface and solid geometries. (C) The Amira 3-D segmentation editor can be used to seed and label particular voxels that correspond to chosen plant cells within the confocal data set. The use of a specific cell wall stain allows for the easy selection of the internal volumes of individual cells. A closed triangulated surface can be formed over the selected group of voxels using a marching cubes algorithm. (D) Surface-rendered cells are displayed at the correct position and scale within the data set. Each cell has been transformed from an unconnected group of voxels to a single geometric object that can be manipulated at will. Repeating the process builds an accurate representation of the shapes, arrangement, and connectivity of cells within the tissue. 


\section{3-D CELL INTERACTIONS}

Why are plant cell arrangements so interesting? The genetic control of plant development is mediated by cellular interactions, and an exchange of positional information contributes to the self-organization and coordination of cells during development. Developing tissues or organs generally demonstrate a capacity for self-organization. For example, wounded tissues generally respond in a robust and coordinated fashion to allow repair, and local induction events can initiate prolonged and coordinated developmental processes. These types of developmental plasticity and functional autonomy are particularly evident in plant tissues.

The basic features of a plant's body plan are established during embryogenesis, but its final form results from the continued growth of meristems and the formation of organs throughout its life, often in a modular and indeterminate fashion. Plant cells are constrained by rigid cell walls and are generally nonmotile, so there is the clear possibility that cell fates within a meristem are determined by lineage. However, evidence from plant chimera and wounding studies have demonstrated a more important role for cell-to-cell interactions during fate determination (10). It is likely that positional information during plant development is exchanged between the cells, and the fate of cells within a developing tissue is determined by a network of local cellular interactions. To dissect such a network, it is crucial that we can clearly map individual cells and their neighbors inside intact meristems and have the means to manipulate them.

\section{3-D SEGMENTATION}

Segmentation techniques similar to those used in medical imaging can be applied to 3-D confocal data sets. This involves the selection and labeling of particular voxels that correspond to a 3-D object of interest. In the clinician's case, this might correspond to a patient's organ. In our case, this will be a plant cell. Various techniques are available for the selection of volumetric objects, which range from the fully manual to automatic tools that detect volume boundaries or local intensity or texture. We have chosen to use a specific plant cell wall stain that outlines every cell in the tissue (Figure 2). This is very helpful because it allows for the use of automatic segmentation tools to determine the interior volume of a chosen cell. We routinely use Amira ${ }^{\mathrm{TM}}$ (Visual Concepts $\mathrm{GmbH}$, Berlin, Germany), a general purpose physical modeling and data visualization tool. The software provides an interface for visualizing large multidimensional confocal microscopy data sets. For example, the data set shown in Figure 3 consists of $736(512 \times 512)$ optical sections spaced at $0.2 \mu \mathrm{m}$, or $147 \mu \mathrm{m}$ deep. Amira provides a very useful set of input/output, datahandling, and visualization modules and allows software routines to be combined in a modular fashion. This allows for the specialized 3-D reconstruction and visualization techniques to be applied in a flexible way to different types of data, including confocal data sets. The program provides a simple interface, sophisticated, fast-visualization routines, is affordable and robust, and is suitable for both high-end personal computer and UNIX ${ }^{\circledR}$ (The Open Group, Berkshire, UK) hardware.

High-resolution confocal data sets can be rapidly segmented to allow for the direct visualization of cell arrangements within intact plant embryos and meristems. The large data files, up to $250 \mathrm{MB}$, can be directly rendered to allow for excavation of the data, production of sections in arbitrary planes (Figure 3A), and rendering of surface features (Figure 3B). In practice, cells are generally chosen by placing a seed point manually within the center of a cell in the confocal data set. The seed is then inflated in 3-D to find the boundaries of the cell, which are marked by a high intensity of staining. The program provides a segmentation editor for this purpose (Figure 3C). The exterior geometry of a segmented cell can then be described using a marching cubes algorithm that, if needed, can be converted to a solid geometry for computer-assisted milling of 3-D models or finite element analysis (Figure $3 \mathrm{D})$. We can now routinely reconstruct the cellular structure of entire meristems for various experiments (Figure 4).

We can use these computer visualization methods, adapted from the medical imaging field, to reduce large data sets to a much more compact and simple description of the 3-D shapes and relative arrangement of cells in a meristem. Because cell-to-cell signaling plays such an important role in plant development, these techniques show much promise for the analysis of genetically perturbed plants and as a basis for modeling the cellular interactions themselves.

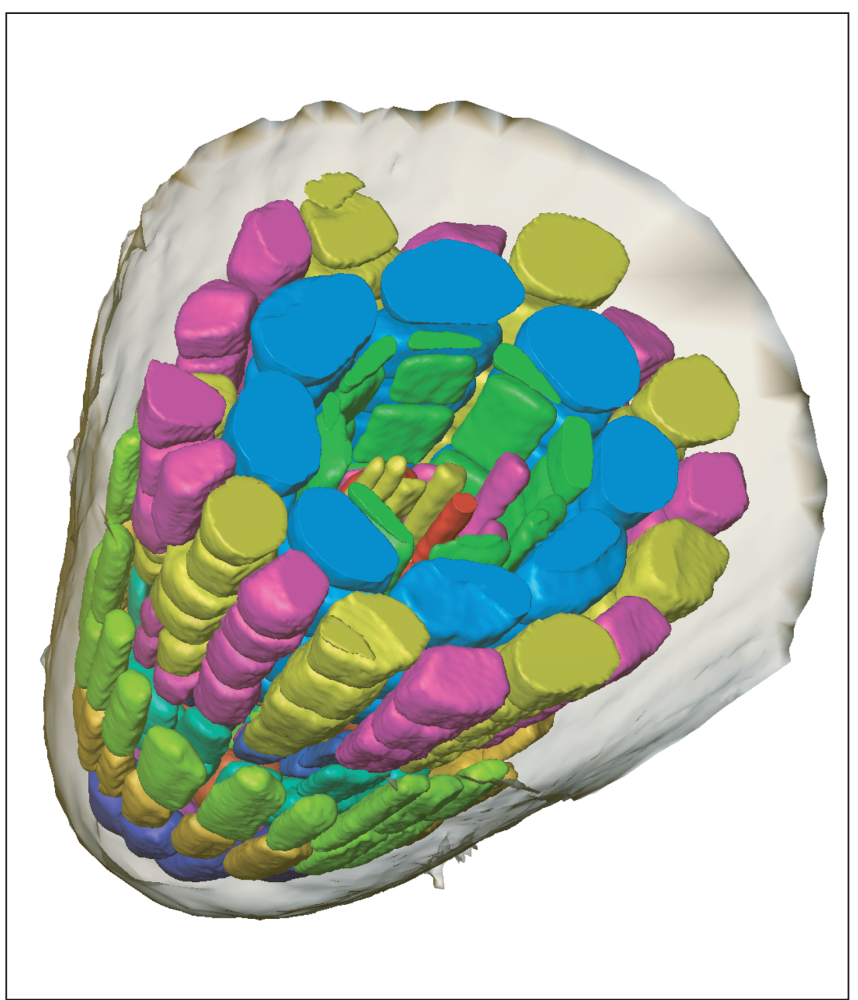

Figure 4. Organization of the Arabidopsis root meristem. 3-D segmentation allows for the complete description of cellular organization of Arabidopsis root meristems. Individual cell types can be labeled and subjected to virtual dissection using 3-D visualization software. 


\section{GENETIC MANIPULATION AND MODELING}

To dissect local cell-to-cell interactions, it is crucial that we can clearly visualize individual cells inside living meristems and have the means to perturb them. The combined techniques of classical botanical histology and modern confocal microscopy allow us to visualize 3-D cell arrangements with unprecedented clarity. Molecular genetic tools allow us to manipulate them. In conjunction with the work described earlier, we have devised a complementary scheme for targeted gene expression that is based on the yeast GAL4 transcription activator (11). The system allows us to genetically manipulate cells during meristem development. For example, we have demonstrated the use of GAL4-targeted misexpression to genetically kill specific meristem cells using diphtheria toxin A chain during root meristem development. The combined genetic and visualization tools are providing powerful new methods to determine the rules for cell-to-cell interactions within developing plants.

The new 3-D visualization techniques allow for the construction of a precise and simplified geometric description of the cellular architecture of embryos and meristems. This description could be further reduced to a simple numerical scheme for describing the 3-D organization of cells in the meristem. For example, each cell's position, approximate 3$\mathrm{D}$ shape, and location of adjoining cells could be described in a simple notation, and there is a compelling reason for pursuing this. The fate of an individual plant cell in a meristem is governed by local positional information. Each cell is part of a self-reinforcing network of interactions. To understand the normal regulation of meristem development and mutant defects, we need to turn to dynamic models that incorporate some of these intercellular signaling features. These will be computational models and will need to include $(i)$ an engine for processing the predicted interactions between cells and (ii) a scheme for representing the consequences for cell proliferation and differentiation in the meristem.

Here I've described the novel combination of modern confocal microscopy and computer-based 3-D reconstruction techniques with classical staining and clearing techniques for imaging intact plant tissues rather than thin sections. This allows for the 3-D arrangement of cells in a plant meristem to be captured and potentially converted into a simple numerical form. The application of rules for plant cell interaction to such a model would result in dynamic behavior that could be animated and visualized. In the future, this could be a framework for directly modeling cell-to-cell dynamics in this complex system.

\section{ACKNOWLEDGMENTS}

Thanks to Sue Bougourd, Jo Marrison, and Carol Wenzel, University of York; John Runions, University of Cambridge, for shared interest and discussions; and Barbara Botrill, University of Cambridge, for help in obtaining sectioned material. I wish to thank the Gatsby Charitable Foundation and the Biological and Biophysical Scientific Research Council for financial support for this work.

\section{REFERENCES}

1.Hooke, R. 1961. Micrographica, facsimile ed. Dover Publications, New York.

2.Amos, B. 2000. Lessons from the history of light microscopy. Nat. Cell Biol. 2:E151-152.

3.Harris, H. 1995. The Cells of the Body. CSH Laboratory Press, New York.

4.Mazzarello, P. 1999. A unifying concept: the history of cell theory. Nat. Cell Biol. 1:E13-E15.

5.Titford, M. 1993. George Grubler and Karl Hollborn: two founders of the biological stain industry. J. Histotechnol. 16:155-158.

6.White, J.G., W.B. Amos, and M. Fordham. 1987. An evaluation of confocal versus conventional imaging of biological structures by fluorescence light microscopy. J. Cell Biol. 105:41-48.

7.Ruzin, S.E. 1999. Plant Microtechnique and Microscopy. Oxford University Press, Oxford, UK.

8.Mason, W.T. Fluorescent and Luminescent Probes for Biological Activity. Academic Press, New York.

9.Bougourd, S., J. Marrison, and J. Haseloff. 2000. Technical advance: an aniline blue staining procedure for confocal microscopy and 3D imaging of normal and perturbed cellular phenotypes in mature Arabidopsis embryos. Plant J. 24:543-550.

10.Scheres, B. 2001. Plant cell identity. The role of position and lineage. Plant Physiol. 125:112-114.

11.Haseloff, J. 1999. GFP variants for multispectral imaging of living cells. Methods Cell Biol. 58:139-151.

Address correspondence to Dr. Jim Haseloff, Department of Plant Sciences, University of Cambridge, Downing Street, CambridgeCB2 3EA,UK.e-mail:jh295@cam.ac.uk
Suggestions for contributions to the BioImaging
feature are welcomed by its editor, Dr. Steve
Paddock(paddock@facstaff.wisc.edu) 